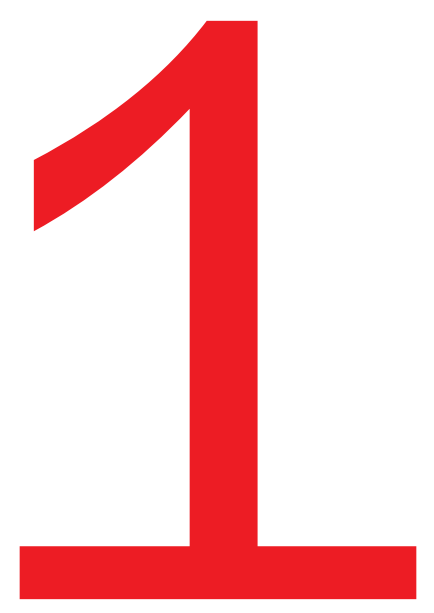

\title{
Résumé
}

Comment les trajectoires de soins des jeunes qui sont psychiatrisé-e-s influencent-elles leur transition à la vie adulte au Québec ? Les jeunes passent maintenant à l'âge adulte dans un contexte social et économique caractérisé par une croissance du travail non régulier et la transformation de la structure familiale. Nous argumentons que ce passage des soins pour enfants aux soins pour adultes vécu par certain-e-s, vulnérabilise cette population déjà marginalisée en exacerbant les défis qu'elles et ils vivent à travers cette transition. Notre analyse d'entretiens menés avec des intervenant-e-s qui travaillent avec ces jeunes montre que leurs trajectoires de soins exacerbent au moins en partie les défis auxquels elles et ils font face. C'est une réalité que nous observons notamment au niveau de leur accès au logement, de leur recherche d'emploi et de leur capacité à construire des projets étalés dans la durée. Nous discutons la façon dont ces expériences influencent la recherche de stabilité et la recherche d'autonomie propre à leurs parcours de vie.

Mots-clés jeunesse, parcours de vie, santé mentale, trajectoire de soins, transition à la vie adulte

\section{Psychiatrisation et transition à l'âge adulte : La perspective des intervenant-e-s}

\author{
ALEXIS H TRUONG, GENEVIÈVE NAULT \& \\ KATHARINE LAROSE-HÉBERT
}

\section{Introduction}

La transformation des besoins des jeunes Québécois-es et des défis liés aux enjeux de santé mentale pour cette population est une question qui préoccupe plusieurs acteur-e-s dans cette communauté. Plusieurs initiatives visent d'ailleurs à accroitre la sensibilité du public à cet égard et à nourrir les débats publics dans un contexte de transformations sociales et économiques plus larges au Québec. Un exemple d'une telle initiative est celui du Mouvement jeunes et santé mentale qui dénonce notamment la difficulté d'accès aux services, la surmédicalisation des problèmes sociaux des jeunes et le besoin d'inclure davantage les jeunes dans l'élaboration de projets propres à des enjeux qui les concernent.[1]

Dans cet article, nous présentons les résultats d'une analyse sur la transformation des défis qui caractérisent l'expérience des jeunes adultes psychiatrisé-e-s au Québec au moment de leur transition à la vie adulte à travers le témoignage d'intervenant-e-s qui travaillent avec cette population. Notre attention est portée sur le processus de psychiatrisation et son effet sur la vie des jeunes, âgé-e-s entre 18 et 35 ans,[2] qui le subissent. Notre questionnement émerge de demandes formulées par des acteur-e-s dans la communauté et d'observations effectuées au cours d'un projet de recherche antérieur, mené par une des cochercheures. Ce projet, qui portait sur les parcours de soins et l'expérience subjective des usagers de services de santé mentale au Québec, a permis d'identifier la particularité des difficultés d'insertion sociale vécues par les jeunes adultes psychiatrisé-e-s et la pénurie des services sociaux et de santé leur étant destinés.[3] Une limite de cette étude est toutefois de ne pas avoir pu étudier 
en profondeur la façon dont ces expériences se construisaient à travers les différents moments du parcours de vie des jeunes en question, dont le passage à la vie adulte.

Plusieurs chercheur-e-s s'entendent pour dire que le passage à la vie adulte est maintenant marqué par davantage d'incertitudes entre autres liées aux questions identitaires, à la recherche d'autonomie, aux responsabilités accrues et à l'employabilité.[4] Pour les jeunes faisant face à des problématiques de santé mentale, la période de transition à la vie adulte s'accompagne d'épreuves supplémentaires tels que le transfert à des services aux adultes peu adaptés à leurs réalités en mouvement, le diagnostic, la prise de médication et la gestion des effets secondaires leur étant associés et la surveillance institutionnelle.[5-9] Or, l'effet des trajectoires de soins propres à cette problématique sur le parcours de vie des jeunes au moment de leur passage à la vie adulte demeure peu étudié.

Comment la trajectoire de soins des jeunes psychiatrisée-s affecte-t-elle les défis qu'elles et ils vivent et les besoins qu'elles et ils identifient au cours de leur transition à la vie adulte ? Voilà la question à laquelle nous tentons de répondre. Nous verrons comment la trajectoire de soins associée à la psychiatrisation des jeunes constitue un facteur de risque et de vulnérabilisation supplémentaire au cours de la transition à la vie adulte qui amplifie encore à ce jour les défis normalement vécus par les jeunes au cours de cette période. Nous définissons les trajectoires de soins à partir de leurs dimensions objectives et subjectives,[10] se construisant à la fois par la suite des positions sociales que les jeunes concernée-s viennent à occuper quant à leurs enjeux en santé mentale mais aussi par la façon dont elles et ils mettent en mot cette expérience lors de leurs rencontres avec les intervenant-e-s que nous avons rencontré-e-s.

Les données que nous avons analysées proviennent d'entretiens menés en 2016 et 2017 avec sept intervenante-s œuvrant dans le domaine de la santé mentale au Québec. Nous avons choisi de travailler avec des intervenant-e-s pour répondre à notre question car ce sont elles et eux qui deviennent en quelque sorte le " pont " entre les pressions sociales et institutionnelles propres aux soins et la façon dont les jeunes concerné-e-s expriment comment elles et ils vivent ce processus. Cinq intervenant-e-s rencontré-e-s provenaient du milieu communautaire et deux travaillaient dans le milieu institutionnel dans les régions de Montréal et de l'Outaouais. Nous inspirant de l'approche des parcours de vie, nous présentons ici une typologie des besoins et des défis identifiés de manière inductive à travers notre analyse des entretiens.[11]

\section{Jeunesse et transition à l'âge adulte}

Qu'est-ce que la "jeunesse " et que signifie " devenir adulte " pour les jeunes, pour une société ? Une explication sociologique longtemps partagée a été que l'entrée dans la vie adulte pouvait être définie à partir de trois critères liés à des changements fondamentaux de statut : "le début de la vie professionnelle, le départ de la famille d'origine et le mariage ".[12] Or, depuis la fin des années 90, nombre de chercheur-e-s constatent un allongement de la période de jeunesse, lequel retarde du même coup l'inscription dans la vie adulte par rapport aux générations précédentes.[13] Les frontières délimitant l'âge de cette population légalement majeure mais ne possédant pas les attributs habituellement reconnus aux adultes[15] s'étalent et varient d'une recherche à l'autre.[16] Dans un rapport publié en 2007 sur le développement de politiques pour le Gouvernement du Canada, Gaudet précise que la jeunesse est difficile à définir puisque les limites pour passer de l'enfance à l'adolescence ou de l'adolescence à l'âge adulte se construisent à partir de nombreux facteurs, dont " les rites symboliques, les évènements de vie, les lois, les normes et les rôles sociaux ".[17,18] À la fin de l'adolescence se rattache ainsi cette " nouvelle " étape de la vie, un phénomène qui a mené à la création de tout un vocabulaire pour désigner cette période transitoire aux caractéristiques particulières, dont l'" emerging adulthood "[19] et l'" adulescence "[20].

Tout comme l'adolescence, et quel que soit le nom utilisé pour la désigner, cette période qui marque l'entrée dans la vie adulte est maintenant elle aussi comprise comme une période critique du développement psychosocial des individus. À tout le moins, cette transition implique un investissement important dans la construction identitaire, la définition des projets de vie et la recherche d'autonomie. [21] Parmi les changements qui touchent la vie des jeunes, on compte notamment l'individuation par rapport à la famille, l'exploration de nouveaux intérêts et de nouveaux loisirs, le développement des habiletés, la découverte de l'intimité et de la sexualité, la formation et la consolidation des relations sociales, le choix de la carrière et le début d'un emploi ou des études supérieures.[22] C'est entre autres à travers l'étude de ces transformations que l'approche des parcours de vie a permis de mieux comprendre comment le contexte dans lequel les jeunes grandissent influence désormais leurs différentes trajectoires et leur expérience de ces moments critiques. Ces travaux nous ont dès lors aidé-e-s à reconnaitre plus facilement les défis auxquels les jeunes font face dans leur parcours, compris comme de véritables enjeux de société.

Toutefois, l'étude des défis particuliers vécus par les 
jeunes adultes psychiatrisé-e-s demeure une limite des recherches sur la transition à la vie adulte. Les études sur la transformation des parcours de vie se sont principalement intéressées aux trajectoires propres à l'éducation, la famille et l'accès au travail régulier. De plus, lorsque des facteurs de marginalisation sont considérés, l'attention a plutôt été portée vers des rapports sociaux de sexe et de genre, de classe ou liés à l'ethnicité.[23] En revanche, la particularité des besoins des jeunes adultes psychiatrisé-e-s et l'importance d'ajuster les services sociosanitaires (tels que le logement, la sécurité alimentaire, la santé, les loisirs et l'employabilité) à leurs besoins ont mainte fois été constatés dans le milieu de l'intervention et dans le milieu de la recherche. II est ainsi surprenant qu'une telle problématique n'ait pas fait l'objet de recherches plus approfondies sur l'effet de ces expériences sur le parcours de vie des jeunes dit-e-s plus vulnérables.

\section{Jeunes vulnérables et psychiatrisation}

L'expérience du passage à la vie adulte se construit en rapport aux normes et aux scripts sociaux qui définissent, dans une société donnée, ce qu'est être un-e adulte et ce qu'est une vie réussie. Les défis que ce rapport aux normes soulève ne sont pas uniformément répartis et participent parfois à marginaliser et à exclure davantage certains groupes. L'individu contemporain doit notamment posséder certaines caractéristiques pour s'inscrire et être valorisé dans les sociétés occidentales : être autonome, responsable et faire preuve d'initiative.[24] Nombre d'études montrent aussi qu'il existe des enjeux particuliers vécus par les jeunes considérée-s " vulnérables " au moment de leur transition à la vie adulte.[25] Beaucoup d'attention est notamment portée aux difficultés rencontrées par les jeunes ayant des trajectoires impliquant les services de la protection de la jeunesse.[26-28]

Plusieurs enjeux liés à la santé mentale coïncident avec la période de transition à la vie adulte. Comme le soulignent Ouellette-Plamondon et coll., la majorité des enjeux de santé mentale débutent avant l'âge de 35 ans.[29] De façon concomitante, les jeunes psychiatrisé-e-s doivent souvent mettre en berne leurs projets scolaires, professionnels et interpersonnels pour centrer leur vie quotidienne sur le contrôle des symptômes et la gestion des effets secondaires.[30] Comme l'indique Parron, les troubles psychiques " perturbent [...] le cadre et les étapes habituels du passage à l'âge adulte ".[31] Ce phénomène va donc à l'encontre de l'expérience des autres jeunes de leur âge et des normes véhiculées par la société,[32] les plaçant malgré eux dans une position de déviance.

Déjà en 1988, Gagné et Poirier signalaient que la psychiatrisation des jeunes entraînait le risque de compliquer leur insertion dans la vie active.[33] Comme Fleury et Grenier[34] le constatent, " [I]es troubles psychiques en-travent spécialement la transition des jeunes à l'âge adulte, puisqu'ils nuisent à l'accès aux études et au marché du travail ainsi qu'aux possibilités d'établir des relations sociales et intimes enrichissantes ". C'est au cours de la transition à la vie adulte que les jeunes actualisent la place qu'elles et ils souhaitent occuper dans la société en investissant divers espaces sociaux tels que la famille et le monde professionnel.[35] Or, les jeunes psychiatrisé-e-s sont souvent contraint-e-s d'investir des relations accroissant la dépendance. La nature de la psychiatrisation augmente souvent leur dépendance à l'institution, à la médication et à la relation thérapeutique avec le personnel soignant. Ces défis, liés au fait de vivre avec un diagnostic psychiatrique, amplifieraient ainsi les exigences qui découlent des transformations sociales et économiques plus larges et qui pèsent sur les jeunes en général.

Ces problèmes ne sont pas nouveaux. Dès 1982, Beau identifiait déjà la problématique du manque de services adaptés et dénonçait le manque d'études sur l'offre de services destinés aux jeunes adultes psychiatrisé-e-s.[36] Selon lui, ces jeunes se retrouvaient dans une structure destinée aux adultes plus âgés et souvent incompatibles avec les besoins particuliers des jeunes. En 1988, Gagné et Poirier relevaient aussi le caractère problématique des pratiques d'insertion sociale destinées aux jeunes psychiatrisé-e-s, exhortant les milieux à créer des services d'interventions alternatives.[37] En 1996, Leclerc considérait que le syndrome de la " porte tournante " était un des signes que le soutien professionnel offert aux jeunes était inadéquat.[38] Plus récemment, Ouellette-Plamondon et coll. soulignent l'absence de services appropriés pour les jeunes, notamment au niveau du soutien quant à la détresse et l'effet de ces enjeux sur leur construction identitaire, leurs projets de vie et leurs relations interpersonnelles.[39]

De façon plus large, plusieurs instances gouvernementales insistent désormais sur la nécessité d'agir auprès de cette population. Selon les stratégies ontarienne et canadienne en santé mentale,[40,41] une des priorités actuelles est la transition des services pour enfants et adolescents vers les services aux adultes. Du côté du Québec, les Plan d'action en santé mentale (PASM) 2005-2010 identifiaient aussi la planification de la transition des services destinés aux jeunes et ceux réservés aux adultes comme un des objectifs prioritaires du Ministère de la Santé et des services sociaux, réitéré dans le PASM 2015-2020.[42,43] L’idée qu'il faudrait développer une offre de services favorisant une continuité 
de soins pour cette population socialement vulnérabilisée semble donc faire consensus relatif, même si elle ne semble pas encore avoir été mise en pratique.

En tant que point tournant dans la trajectoire de soins, le passage des services psychiatriques pour les enfants et les adolescent-e-s à ceux offerts aux adultes est souvent difficile et marqué de ruptures dans l'ensemble des services.[44] Plusieurs auteur-e-s signalent que le manque d'intégration des services et leur incapacité à prendre en charge cette transition est problématique.[45-47] Bien que le taux d'accès aux services diminue généralement lorsque les jeunes atteignent l'âge de la majorité, cette diminution ne peut pas être expliquée uniquement par un affaiblissement de la demande pour ces services.[48] Une transition difficile entre les services de santé mentale pour jeunes et ceux pour adultes mène aussi souvent à l'abandon de service.[49] Plusieurs auteurs renchérissent que les services destinés aux adultes ne sont tout simplement pas adaptés aux besoins des jeunes adultes et sont donc inefficaces pour ces derniers.[50,51] Ces services pour adultes ne répondraient pas aux intérêts des jeunes, ne leur offriraient ni le soutien nécessaire à cette étape de leur parcours, ni la possibilité d'interagir avec des pairs du même âge et agirait souvent comme un accompagnement insuffisant ou trop encadrant.[52] Or, comme l'indique Gaudet, "[I]a période de semi-autonomie qui caractérise les jeunes adultes fragilise particulièrement ceux qui n'ont pas de réseau de soutien [adéquat] ".[53]

\section{La quête de stabilité}

Selon les intervenant-e-s que nous avons rencontré-e-s, la recherche de stabilité est complexifiée pour les jeunes psychiatrisé-e-s par le désir ou la pression qu'elles et ils ressentent à "gérer " leur santé mentale. Le défi que représente ce travail sur soi est notamment amplifié par le fait qu'il passe plus souvent qu'autrement par l'obtention de services de santé mentale et sociaux qui sont eux-mêmes difficiles à naviguer. Nous identifions ainsi trois façons dont l'accès problématique aux soins mine invariablement la capacité des jeunes adultes psychiatrisé-e-s à " stabiliser " leurs situations de vie à travers leur trajectoire de soins, trajectoire qui entraine et désorganise parfois à son tour les autres trajectoires socioéconomiques à travers lesquelles ces jeunes naviguent.

Premièrement, comme l'explique un de nos répondants, l'accès aux services spécialisés au Québec passe nécessairement par la référence, souvent conditionnelle à l'accès à un médecin de famille qui " ouvre des portes " dans un contexte institutionnel où l'accès aux médecins de famille demeure très difficile. "Être sur une liste d'attente " devient une histoire commune dans un système où tout est centralisé. Certain-e-s intervenant-e-s expliquent qu'" il n'y a plus de contact humain ", créant une situation "déshumanisante " pour les jeunes qui cherchent des services. L'état des services de santé et sociaux est d'autant plus alarmant pour les jeunes qui ont eu leur premier épisode de crise avant l'âge de la majorité, puisqu'il existe une coupure de service pour jeunes à l'âge de 18 ans. Or, aucun mécanisme réel n'est mis en place pour assurer cette transition des services pour jeunes à ceux destinés aux adultes. Comme une de nos répondantes l'explique, le transfert n'implique pas nécessairement de suivi et ne se fait pas automatiquement lors de la transition, et c'est la personne ou ses proches qui doivent " prendre en charge " cette importante étape de la trajectoire de soins. Ceci devient souvent une expérience " anxiogène " pour ces jeunes et leur famille et qui se compile aux autres défis qu'elles et ils affrontent. Un répondant explique ainsi que la personne doit de fait fonctionner sans soutien réel jusqu'à sa prochaine difficulté. Selon lui, il est impératif de créer des espaces où les jeunes pourraient présenter plusieurs demandes, sans être référés " à gauche et à droite ", où il y aurait un soutien et un accompagnement pendant le référencement.

Plus largement, du point de vue des intervenant-e-s à qui nous avons parlé, le manque de ressources financières et de ressources humaines exacerbe ce problème d'accès aux services en atrophiant la quantité, la diversité et la qualité des services. Les services propres à la prévention des crises sont mis de côtés pour se concentrer sur les situations d'urgence comme celles liées à l'hospitalisation et à la garde en établissement. Un intervenant explique que :

On voit souvent que les gens crient, crient, crient à l'aide, mais les soins arrivent quand y'est trop tard, quand c'est la crise, crise suicidaire, homicidaire. Là on judiciarise. Tout ce processus-là. Arrive à l'hôpital, bon y'a la garde en établissement, toutes les conséquences qui déboulent.

Pour les personnes qui vivent leurs premiers épisodes de crise, ceci signifie aussi que les ressources sont investies dans l'évaluation et non pas dans les suivis qui se raréfient. Or, les interventions préventives ou tôt dans la trajectoire de soins ont un effet positif à long terme et aident parfois à prévenir de nouvelles hospitalisations. Malgré les objectifs en santé mentale définis dans le plan stratégique du Ministère de la Santé et des services sociaux, force est toutefois de constater que le système actuel finit souvent par offrir des soins lorsque les situations deviennent " urgentes " ou dans des situations de crise, et ce au détriment des patient-e-s.

Un tel état de choses contribue non seulement à marginaliser mais aussi à vulnérabiliser les jeunes adultes psychiatrisé-e-s à travers une trajectoire de soins qui devient parfois pour elles et eux une épreuve vécue en marge des services promis. Au Québec, le fonctionnement " par épisode " des traitements complexifie aussi l'accès véritable aux soins car la nature parfois imprévisible des rechutes se conjugue mal à cette approche non continue qui ne tient pas compte ni de l'évolution 
de la situation de vie de ces jeunes ni de leur développement au cours de cette période cruciale qu'est le passage à la vie adulte. Comme une intervenante rencontrée l'indique : "C'est très laborieux comme cheminement pour avoir accès aux soins d'autant plus qu'ils sont discontinus, insuffisants... en tout cas, ça marche pas! "Une autre personne ajoute que ce sont d'ailleurs "ces services épisodiques " qui font en sorte que les suivis sont " toujours à recommencer et que les personnes ne se sentent pas aidées. "Ainsi, sachant que la transition à la vie adulte est réputée amener son lot de défis - notamment au niveau de l'insertion sociale et de la sécurité économique -, il semblerait judicieux de considérer comment ces facteurs de précarisation sociale et économique participent à fragiliser la situation de vie de ces jeunes adultes qui affrontent des enjeux en santé mentale.

Deuxièmement, la rigidité des mandats et l'organisation des programmes autour de clientèles cibles ont parfois l'effet pervers de restreindre l'accès aux soins, en particulier dans le réseau public. Certain-e-s intervenant-e-s expriment ainsi souvent entendre des explications comme : " désolé, elle [n'est pas éligible], on ne peut pas l'aider. " Une autre intervenante exprime :

On est dans ce régime-là très, très rigide de s'en tenir à notre mandat respectif et à travers les trois lignes, première, deuxième, troisième ligne c'est tout le temps de même. Fait que les services se lancent la balle. Ah non c'est pas à nous de faire ça, va voir tel autre service. Ah non c'est pas à nous non plus, va voir l'autre service. Fait que là le jeune ou sa famille se retrouve tout seul puis ne savent plus où se tourner parce que tout le monde leur ferme la porte.

Un intervenant soulève que ce type d'enjeu est moins prononcé dans le milieu communautaire, qu'il dit être plus " à l'affut de besoins émergents ". La situation est toutefois compliquée par le manque de communication et de coordination entre les différents services de santé et services sociaux, qu'ils soient de première, deuxième ou troisième ligne. Une intervenante ajoute que :

C'est la défaillance des services. C'est la défaillance de l'organisation des soins. La défaillance de, mon dieu comment j'peux dire ça? Y'a pas d'arrimage. Ça travaille en silo. Chacun fait son affaire à sa façon sans consulter les autres. [...] J'finis juste par mettre un plaster sur le bobo.

Même lorsque les jeunes parviennent à accéder à des services, ces services se conjuguent souvent mal à la diversité des besoins exprimés par les jeunes adultes, à la complexité des situations qu'elles et ils vivent et à la dimension continue de leur trajectoire de soins. Ces jeunes sont ainsi appelé-e-s à faire concorder leurs expériences individuelles complexes avec la "grille officielle ", ce qui ne leur garantit pas pour autant des services ajustés à leurs besoins. De façon large, les services destinés aux adultes ne sont pas toujours bien ajustés aux besoins des jeunes. Plusieurs trouvent ainsi que " le milieu est pas fait pour eux".

Finalement, nos répondant-e-s dénoncent ce qu'elles et ils voient comme un besoin d'intégrer les jeunes dans les prises de décision quant à leurs soins. De façon large, le système de soins est perçu comme ayant une interprétation " très rigide " de la loi sur la confidentialité, ce qui limite la consultation des familles et les mettent à l'écart - bien que ce soit souvent sur elles et eux que repose le travail de pallier les lacunes du système. Un intervenant exprime : "On informe très peu les gens des services qui sont disponibles et comment ils fonctionnent. Les personnes n'ont pas le vocabulaire du système, on doit leur montrer quels mots utiliser pour nommer leurs difficultés afin d'obtenir des services. "Or, ce n'est pas seulement un manque d'information. On voit parfois les contours d'une culture qui ne définit pas ces jeunes comme des acteur-e-s légitimes dans leur traitement ou comme étant en droit de signifier leurs besoins ou leurs désirs quant au traitement - en particulier lorsque ces positions entrent en conflit avec les discours médicaux dominants quant aux traitements jugés favorables. Ainsi, une intervenante exprime:

On ne les tient pas trop au courant de leurs droits pis ça c'est volontaire. On leur dit pas trop de choses. On les tient dans l'ignorance. Moi ça me choque. Nos jeunes qui sont en garde en établissement ben au lieu de leur faire part de tous leurs droits, de leur expliquer de façon très éclairée ce qui en est, on leur dit pas. Même qu'un moment donné [un-e membre des équipes de soins] est allé-e chercher un dépliant et a remis ça au jeune, qui a tout expliqué : t'as le droit de contester, t'as le droit de ci, ça, ça pis [cette personne] s'est fait pointer du doigt par toute l'équipe [...] Pis voyons t'es qui toi pour aller lui dire ça, t'es à contre-courant avec ce que l'équipe a décidé, blah, blah, blah. Quand en fin de compte [cette personne-là] tout ce qu'[elle] voulait faire c'est juste faire part des droits du jeune par rapport à la situation qu'il vivait, par rapport à sa garde en établissement.

La psychiatrisation n'est pas qu'un simple stigmate : ce processus rend parfois les jeunes " otages " de leur trajectoire de soins en imposant des façons de prendre soin de soi comme plus légitimes et plus valorisées que d'autres, lesquelles deviennent étiquetées comme problématiques par certaine-s professionnel-le-s, par la société. De plus, il arrive parfois que certain-e-s professionnel-le-s de la santé aient aussi leurs propres préjugés envers la santé mentale ou qu'elles et ils doivent négocier des tensions au sein de leur équipe de travail - réalités susceptibles d'avoir des conséquences négatives pour les jeunes concerné-e-s. 


\section{La quête d'autonomie}

La seconde catégorie de besoins qui émerge de notre analyse des entretiens avec les intervenant-e-s est celle de la recherche d'autonomie qu'éprouvent les jeunes avec qui ces intervenant-e-s travaillent. Ce désir d'autonomie est une question qui - comme la recherche de stabilité - concerne les jeunes adultes dans leur ensemble.[54] Certain-e-s auteur-e-s identifient d'ailleurs la volonté des jeunes adultes de gérer par eux-mêmes leurs difficultés comme une " barrière " quant à la recherche d'aide en santé mentale.[55] II faut ainsi reconnaitre qu'apprendre à identifier les difficultés en émergence pour savoir quand demander de l'aide et comment l'obtenir est un défi important pour les jeunes adultes en général, ici amplifié pour celles et ceux qui sont psychiatrisé-e-s :

Ces jeunes sont soumis à développer une forme de maturité, on pourrait dire, précoce. Découvrir sa vulnérabilité, savoir ce qui nous pose des difficultés, reconnaitre chez soi c'est quoi, par exemple, les défis principaux. Ça, ça en est un défi pour les jeunes j'trouve parce que je disais tantôt, ils explorent souvent leurs demandes d'aide [...] C'est-à-dire qu'un moment donné faut avoir la maturité de reconnaitre que c'est des cycles, que ça va probablement revenir pis qu'il faut se pencher sur ça, pas juste sur l'anxiété [...] prendre soin de soi, d'une certaine manière.

Largement, " être autonome " fait partie de la norme sociale qui définit les façons d'" être adulte. " Pour ces jeunes, la recherche d'autonomie est donc compliquée par l'expérience de psychiatrisation car elle fait entrer en conflit le désir d'être autonome, présenté comme un trait valorisé, avec le besoin de support et d'aide qui découle souvent des enjeux en santé mentale. Or, de façon plus large, l'image du " bon patient " est souvent interprétée comme celle de la docilité.[56] C'est dans ce contexte d'apprentissage quant à l'" exploration de la demande d'aide " que se compile les enjeux liés à la recherche d'autonomie lors du passage à l'âge adulte, c'est-àdire la décision ou l'obligation de quitter le logement familial, l'accès au logement et la recherche de ressources financières pour devenir autonome financièrement.

Les intervenant-e-s avec qui nous avons parlé soulignent les enjeux entourant la vie dans la maison familiale et le départ de la maison parentale. Le départ de la maison parentale est généralement interprété par les jeunes adultes comme la source d'une plus grande indépendance, mais elle est souvent accompagnée de la perte de soutien social et économique identifié comme significatif chez plusieurs. Une répondante rappelle ainsi que :

Même ceux qui sont pas en difficulté, combien de jeunes à la trentaine, peut-être même après un doctorat, qui viennent revivre chez les parents parce que ben c'est ça, c'est toute la composition sociale, avant de se trouver un emploi, les logements sont terriblement coûteux. La plupart peuvent pas vivre seul en appartement, la plupart vivent en colocation. Pis là on parle même pas de problèmes de santé mentale là-dedans.

Ceci est encore plus marqué chez les jeunes adultes psychiatrisé-e-s qui dépendent du soutien de leur famille, particulièrement quand les services publics ne parviennent pas à remplir leurs besoins. Effectivement, ces soins peuvent demander beaucoup de temps, d'énergie et de ressources financières. Plusieurs répondant-e-s soulèvent ainsi l'importance d'offrir du soutien aux familles ou aux personnes significatives qui interviennent dans la vie de ces jeunes, notamment pour offrir des périodes de répit.

Comme les intervenant-e-s l'indiquent, certaines familles choisissent aussi parfois d'encourager leurs enfants devenu-e-s adolescent-e-s ou jeunes adultes à quitter la maison familiale. Dans certaines situations, des recours judiciaires sont utilisés pour y parvenir. Comme l'indique une intervenante:

Les familles savent plus qui appeler, vers qui se tourner. Ils n'ont pas les services, ils savent pas qui appeler en situation d'urgence pis leur enfant est en train de tout briser dans la maison, de péter les murs fait qu'eux sont démunis, ils savent pas quoi faire, fait que leur dernier recours ben c'est d'appeler la police. Quand ils nous racontent ça, ils se sentent tellement mal. Ils sentent tellement de culpabilité. Ils se jugent, là on est des mauvais parents, on n'a pas été capable de gérer ça.

Bien que ceci peut aussi être la réalité de nombre de jeunes adultes en général, force est de constater une différence quant aux besoins des jeunes adultes psychiatrisé-e-s pour les aider à faire face aux enjeux qu'elles et ils vivent en santé mentale. De plus, dans ces situations où les jeunes doivent se trouver un nouveau logement, les intervenant-e-s rencontré-e-s expriment que l'accès à un logement devient un défi important pour plusieurs. D'un côté, certain-e-s jeunes vivent de la stigmatisation. De l'autre, un logement autonome n'est pas toujours une réelle option pour certain-e-s. Plusieurs répondant-e-s revendiquent ainsi plus de logements supervisés où les jeunes adultes psychiatrisé-e-s pourraient développer leur autonomie tout en recevant différentes formes de soutien.

Un dernier obstacle que nous désirons mentionner au niveau de l'obtention d'un logement autonome et qui a été discuté par les intervenant-e-s concerne l'indépendance financière. $\mathrm{Au}$ Québec, les jeunes adultes qui habitent chez leurs parents et qui n'ont pas de revenus ne sont pas éligibles pour 
l'assistance sociale puisqu'ils habitent chez leurs parents. En revanche, elles et ils ont besoin d'un revenu pour obtenir un logement. C'est un cercle vicieux qui les vulnérabilise. Une intervenante explique ainsi que :

Un jeune qui reste encore chez ses parents, ça peut être difficile pour lui dans les premières démarches pour obtenir de l'aide sociale, pension d'invalidité ou quoique ce soit, c'est très, très, très laborieux pour les parents et ces personnes-là. II y a beaucoup de barrières bureaucratiques.

De façon concomitante, l'entrée sur le marché du travail ou le retour au travail dans une perspective de réinsertion peut signifier la perte de l'assistance sociale qui engendre aussi une perte de stabilité et d'autonomie financière. Si trouver du travail régulier est un défi pour tous les jeunes adultes en général, les obstacles se compilent pour les jeunes qui sont psychiatrisé-e-s, notamment en raison du diagnostic, de la stigmatisation vécue, de la judiciarisation qu'elles et ils vivent parfois et des défis propres à une scolarisation parfois précarisée par les premiers épisodes de crise en jeune âge. Ces enjeux doivent être considérés, même s'ils ne sont pas nécessairement rendus immédiatement visibles ou encore sérieusement considérés par tou-te-s les acteur-e-s impliquée-s lorsque les jeunes se retrouvent en situation de crise ou d'urgence.

\section{Quête de futur}

Dans cette recherche de stabilité et d'autonomie, ce qui dévie de la norme ou qui diffère simplement de cette conception large d'une vie réussie dans l'imaginaire collectif devient un problème à régler. Un intervenant précise que c'est une pression avec laquelle vivent beaucoup de jeunes qui passent à la vie adulte :

Tout le monde dans cette période transitoire, nécessairement, vivent des difficultés. Que ce soit une crise identitaire ou que ce soit une crise d'adolescence, comme on pourrait dire. Et à travers cette période-là, il y a des échecs ou il y a des deuils, des relations qui se forment, qui se déforment, etc., etc. L'expérience avec le monde professionnel, avec l'amour, le sexe, et cetera, et cetera. Pis toutes les expériences négatives, qui sont selon moi, des apprentissages normaux, sont considérés comme des pathologies.

Plusieurs vivent des défis et des difficultés, mais la psychiatrisation comporte aussi ses propres défis, dont nous devons être critiques, et qui peuvent certes influencer de façon parfois négative le parcours des jeunes qui vivent avec des enjeux en santé mentale.

Selon plusieurs des intervenant-e-s rencontré-e-s, la psychiatrisation vient mettre la vie des jeunes avec qui elles et ils travaillent sur " pause ", un "frein " qui les force souvent à abandonner leurs projets de vie à moyen et long terme ou encore à repousser ces projets à plus tard. La psychiatrisation, c'est aussi un " détour ". Or, comme l'indique cette intervenante, les premières expériences sont souvent vécues comme un choc, une expérience violente " assénée " par le système de soins :

Les structures qui sont réputées être bonnes, ou nous protéger, comme l'hôpital, t'es supposé aller te faire soigner. Pis là t'arrives, ils t'enferment contre ton gré, ils te piquent dans la fesse avec des cochonneries que tu ne sais pas c'est quoi pis là tu perds ta job, tu perds ton logement, tu perds ta blonde, t'es plus assurable. Fait que t'es entré là pour te faire aider... je suis assez péjoratif par rapport au réseau de la santé parce que moi, je m'inspire de ce que les gens disent.

Le retour à la famille, aux études ou au travail peut ainsi être marqué par de nombreux défis, dont une stigmatisation quant aux enjeux en santé mentale qui persistent toujours. Une intervenante exprime que plusieurs jeunes adultes avec qui elle travaille lui parlent de devoir " gérer les questions et les regards des autres ". Pour d'autres, c'est tout simplement passer en mode " survie ".

Ces intervenant-e-s nous disent aussi que l'expérience des jeunes qu'elles et ils rencontrent se construit dans un rapport au passé qui est parfois vécu comme un " deuil ", et où les attentes face au futur doivent être "négociées ":

Des fois c'est pas tant les gens qui parlent de leurs perspectives à long terme, c'est plus qu'ils reflètent le deuil de leur ancienne vie. C'est, avant qu'il m'arrive ça j'étais dont comme ça, j'étais capable de faire ci, j'étais capable de faire ça. Maintenant je suis comme ça. [...] C'est un deuil. C'est comme si leur maladie ou l'abus, l'injustice qui est arrivé c'est hors de leur contrôle ce qui fait que toute leur vie est chamboulée à cause de ça. [...] Oui les gens aspirent à retourner là, mais [avec] le rétablissement souvent t'arrives pas au même point qu'avant.

Comme l'explique cet intervenant, les attentes des jeunes ne sont pas toujours compatibles avec leur nouvelle réalité, la façon dont les soins sont organisés ou encore la façon dont leur trajectoire de soins se développera au cours des années à venir. II y a "rêve de guérison " et ceci est une source de motivation qui donne aux jeunes " une raison de se lever le matin ", mais il reste que plusieurs d'entre elles et eux devront vraisemblablement vivre avec ces enjeux pour la durée de leur vie.

De plus, les rêves et les aspirations des jeunes sont aussi influencés par ce qu'elles et ils viennent à définir comme " la norme ". Un autre intervenant explique ainsi que :

[Ces jeunes adultes] se mesurent surtout par des rôles institués : être un père, être un travailleur, être une mère, avoir un emploi, avoir une maison. C'est là que 
le normatif entre en ligne. Les gens sont beaucoup en dialogue avec des rôles préconstitués et de l'extérieur, qui peuvent avoir l'air un petit peu, pas futiles, mais désincarnés.

Or, cette projection dans le futur rend invisible les difficultés que cachent ces rôles, en particulier dans le contexte social et économique actuel. Les jeunes regardent leurs pairs, se comparent à elles et eux et veulent suivre les mêmes étapes.

Or, comme l'explique ce même intervenant:

Souvent il faut venir défaire, j'ai l'impression certains trucs de manière toute délicate. De la même manière que de l'extérieur, ils ont l'impression que leurs amis ont des trajectoires linéaires alors que quand on est dans la norme, quand on est un étudiant je veux dire, il n'y a rien de linéaire. Oui il peut y en avoir, mais donc souvent c'est des jugements qu'on pose de l'extérieur donc eux y se comparent à ce qu'ils voient de l'extérieur et ont l'impression d'être tout à fait en décalage. [...] C'est très bien, mais la manière qu'ils en parlent, c'est justement un peu romancé. C'est comme si aussi ils allaient arrêter de vivre des difficultés.

Ainsi, pour cet intervenant, le travail d'intervention est aussi d'aider ces jeunes à faire coexister les rêves et les difficultés : "les deux sont là, même probablement que les deux vont toujours être là. Pis même pour une personne qui vit pas particulièrement de problèmes sévères de santé mentale, il y a des périodes de vie très difficiles. "

Ceci ouvre la question complexe de définir pour soi et pour autrui ce qui constitue un projet de vie "légitime " ou "valorisable ". À cet effet, un autre intervenant insiste sur le fait que le projet de vie doit refléter les intérêts de la personne et qu'il faut valoriser tout projet de vie, même lorsqu'ils ne sont pas "traditionnels " ou qu'ils dévient de la norme. Un intervenant précise qu'un projet de vie peut être une question de développement de soi ou encore la simple décision d'explorer un intérêt personnel :

Un projet de vie, c'est leur rêve, très important. Oui l'emploi c'est important, oui la médication c'est important, oui pas avoir de consommation de drogue c'est important, mais dépendant la personne, ce qu'elle veut, si la personne veut apprendre la musique ou a un intérêt au niveau de l'art pis elle veut se créer un murale ou avoir un projet au niveau de l'artisanat, au niveau des jeux vidéo, au niveau de leurs intérêts, ben ça peut être un projet de vie juste comme ça. [...] C'est selon leurs intérêts et leurs rêves.

Le projet de vie est donc important au rétablissement, ou du moins à la construction du sens que prend la vie, une vie où la trajectoire de soins occupe à certains moments une place centrale. Force est toutefois de constater que de définir et de réaliser "son projet de vie " est de plus en plus imaginé comme la responsabilité des individus qui doivent " jongler " avec les imprévus de la vie. Or, les jeunes psychiatrisé-e-s sont souvent plus vulnérables quant aux transformations sociales et économiques à plus grande échelle et qui, au cours des dernières décennies, sont venues amplifier la précarité des groupes plus vulnérables, dont les personnes vivant avec des enjeux en santé mentale et les jeunes adultes de façon plus large.

\section{Conclusion}

La majorité des recherches sur la transition à la vie adulte chez les jeunes vivant avec un problème de santé mentale s'intéresse aux difficultés de transition chez les jeunes, sous la responsabilité de la Direction de la protection de la jeunesse ou autres formes d'institutionnalisation, ou cible exclusivement la première psychose. La question du passage à la vie adulte chez les jeunes personnes psychiatrisées et l'offre de services adaptés pour cette population a fait l'objet de peu d'études scientifiques. Encore aujourd'hui, peu d'avancées ont été constatées malgré que les services aux jeunes adultes vivant une problématique de santé mentale soient annoncés comme une priorité par de nombreuses instances politiques. L'expérience de cette population doit être mieux comprise puisqu'elle constitue un groupe particulièrement vulnérable pour lequel les services sont peu ajustés. Notre recherche cherchait ainsi à contextualiser une problématique peu documentée qui affecte plusieurs jeunes adultes psychiatrisé-e-s et qui a des répercussions sur la qualité de vie de plusieurs.

Une transition à la vie adulte, marquée d'épreuves particulières, mène souvent les jeunes psychiatrisé-e-s à une dépendance sur les institutions, la médication et les intervenant-e-s. Cela mine leur insertion sociale en posant obstacle à des projets de vie dits "conformes " aux normes sociales généralement partagées comme le succès dans les études, l'accès à un emploi valorisé ou des relations interpersonnelles stables et satisfaisantes. Ceci contribue à la marginalisation de cette population d'individus. II paraît donc d'une grande pertinence de développer des services spécifiques pouvant soutenir et accompagner les jeunes adultes psychiatrisé-e-s dans le développement de leur identité et de compétences nécessaires à leur intégration au monde adulte - tout en tenant compte de leurs enjeux et besoins particuliers.

Considérant leur rôle pivot, les intervenant-e-s des services sociaux et de santé semblent être dans une position privilégiée pour revendiquer des services continus, intégrés et adaptés aux besoins des jeunes adultes psychiatrisé-e-s. À l'instar de la littérature et des intervenant-e-s avec qui nous nous sommes entretenu-e-s, il semble également d'une 
importance capitale d'inclure les jeunes adultes psychiatrisée-s dans l'élaboration de tels services afin que ces services reflètent leurs besoins et répondent réellement aux défis qu'elles et ils rencontrent. Une piste de recherche future serait ainsi de mener des entretiens directement avec des jeunes de cette population pour mieux comprendre leur perspective et comment ces enjeux s'articulent dans leurs récits.

En explorant la particularité des difficultés d'insertion sociale vécues par les jeunes psychiatrisé-e-s et leur expérience avec l'organisation des soins, cet article a tenté de comprendre certains des besoins identifiés par des intervenant-e-s des milieux communautaire et institutionnel pour mieux soutenir les jeunes avec qui elles et ils travaillent et les accompagner dans cette période transitoire marquée d'épreuves contribuant à leur marginalisation. Des recherches supplémentaires seront nécessaires pour proposer et évaluer de nouveaux outils et services mieux ajustés aux jeunes, comme l'utilisation des médias sociaux aux fins d'intervention. Le milieu communautaire regorge de telles initiatives, trop souvent étouffées par un manque de ressources.

\section{Références}

1.Mouvement jeunes et santé mentale. Déclaration commune [En ligne]. 2017. [consulté le 19 septembre 2018] Disponible : http://mouvementjeunessm.com/revendications-actions.

2.Leclerc C. Adaptation à la communauté de jeunes adultes ayant vécu une ou plusieurs hospitalisations en psychiatrie. Santé mentale au Québec. 1996 ; 212 : 53-72.

3.Larose-Hébert K. Quand les mots se heurtent aux maux : Portait d'un discours morcelé. Étude de l'expérience subjective des personnes utilisatrices des services de santé mentale au Québec [Thèse]. Ottawa (ON) : Université d'Ottawa ; 2016.

4.Voir entre autres : Bidart C. (dir.) Devenir adulte aujourd'hui. Perspectives internationales. Paris : L'Harmattan ; 2006 ; Gaudet S. La responsabilité dans les débuts de l'âge adulte. $2001 ; 46: 71-83$.

5.Davis M et Sondheimer D. State Child Mental Health Efforts to Support Youth in Transition to Adulthood. The Journal of Behavioral Health Services and Research. 2005; 32(1) 27-42.

6.Davis M. Addressing the Needs of Youth in Transition to Adulthood. Administrative and Policy in Mental Health. 2003; 30(6) : 495-509.

7.Davidson S et Cappelli M. We've Got Growing Up To Do: Transitioning Youth From Child and Adolescent Mental Health Services to Adult Mental Health Services. [En ligne]. Ontario Center of Excellence for Child and Youth Mental Health; 2011.
Disponible: http://www.excellenceforchildandyouth.ca/sites/ default/files/resource/policy_growing_up_to_do.pdf.

8.Veilleux $C$ et Molgat $M$. Que signifie être jeune adulte aujourd'hui ? Les points de vue de jeunes adultes ayant reçu un diagnostic de maladie mentale. Reflets. 2010 ; 16(1) : 15279.

9.Gagné J et Poirier M. Formes de l'appauvrissement et insertion sociale des jeunes adultes psychiatrisés. La réinsertion sociale. 1988; 13(1) : 132-43.

10.Dubar C. Trajectoires sociales et formes identitaires. Clarifications conceptuelles et méthodologiques. Sociétés Contemporaines. $1998 ; 29$ : 73-85.

11.Cette recherche a été approuvée par le Bureau d'éthique et d'intégrité de la recherche de l'Université d'Ottawa.

12.Gallant O. Sociologie de la jeunesse. Paris : Armand-Colin ; 2017.

13.Gaudet S. L'émergence de l'âge adulte, une nouvelle étape du parcours de vie. Implications pour le développement de politiques. Document de discussion. [En ligne]. Ottawa: Gouvernement du Canada : Projet de recherche sur les politiques ; 2007 [consulté le 19 septembre 2018]. Disponible: http://horizons.gc.ca/sites/default/files/Publication-altformat/dp_youth_gaudet_200712_f.pdf.

14.Turcotte ME, Bellot C. Vers une meilleure compréhension de la contribution des services sociaux à l'insertion sociale de jeunes adultes en difficulté. Société et jeunesse en difficulté. $2009 ; 8$.

15.Moriau J. Sois autonome ! Les paradoxes des politiques publiques à destination des jeunes adultes en difficulté. Dans : Goyette M, Pontbriand A, Bellot C. Les transitions à la vie adulte des jeunes en difficulté. Québec: Presses de I'Université du Québec ; 2011.

16.Gauthier M. L'âge des jeunes : un fait social instable. Lien social et politique. $2000 ; 43$ : 23-33.

17.Gaudet S. L'émergence de l'âge adulte, une nouvelle étape du parcours de vie. Implications pour le développement de politiques. Document de discussion. [En ligne]. Ottawa: Gouvernement du Canada : Projet de recherche sur les politique ; 2007 [consulté le 19 septembre 2018]. Disponible: http://horizons.gc.ca/sites/default/files/Publication-altformat/dp_youth_gaudet_200712_f.pdf.

18.Elder GH, Kirkpatrick Johnson M, Crosnoe R. The Emergence and Development of Life Course Theory. Dans: Mortimer J et Shanahan MJ. Handbook of the Life Course. s.I : 
Springer ; 2005.

19.Arnett JJ. Emerging Adulthood: A Theory of Development from the Late Teens Through the twenties. American Psychologist. 2000 ; 55(5) : 469-80.

20.Anatrella T. Interminables adolescences. Les 12-30 ans. s.I. : Éditions du Cerf ; 1988.

21.Gaudet S. L'émergence de l'âge adulte, une nouvelle étape du parcours de vie. Implications pour le développement de politiques. Document de discussion. [En ligne]. Ottawa: Gouvernement du Canada : Projet de recherche sur les politiques ; 2007 [consulté le 19 septembre 2018]. Disponible: http://horizons.gc.ca/sites/default/files/Publication-altformat/dp_youth_gaudet_200712_f.pdf.

22.Ouellet-Plamondon C, Dubreucq S, Bérard M, Daneault JG, L'Heureux S, Olivier D et coll. L'intervention précoce pour la psychose... au Québec et à travers le monde. Le Partenaire. 2012 ; 21(1) : 4.

23.Mortimer J et Shanahan MJ. Handbook of the Life Course. s.l.: Springer ; 2005.

24.Ehrenberg A. La Fatigue d'être soi. Dépression et société. Paris : Éditions Odile Jacob ; 2000.

25.Turcotte ME, Bellot C. Vers une meilleure compréhension de la contribution des services sociaux à l'insertion sociale de jeunes adultes en difficulté. Société et jeunesse en difficulté. 2009 ; 8 ; Osgood DW, Foster EM, Courtney ME. Vulnerable Populations and the Transition to Adulthood. The Furture of Children. $2010 ; 20: 1$.

26. Avery RJ, Freundlich M. You're All Grown Up Now: Termination of Foster Care Support at Age 18. Journal of Adolescence. 2009; 32(2) : 247-57.

27.Collin ME, Spencer R, Rolanda W. Supporting Youth in the Transition from Foster Care: Formal and Informal Connections. Child Welfare. 2010 ; 89(1) : 125-43 ; Cunningham MJ, Diversi M. Aging Out: Youth's Perspective on Foster Care and the Transition to Independance. Qualitative Social Work. 2013 ; 12(5) : 587-602.

28.Goyette M, Frechon I. Comprendre le devenir des jeunes placés : la nécessité d'une observation longitudinale et représentative tenant compe des contextes socioculturels et politiques. Revue française des affaires. 2013; 1 : 165-80.

29. Ouellet-Plamondon C, Dubreucq S, Bérard M, Daneault JG, L'Heureux S, Olivier D et coll. L'intervention précoce pour la psychose... au Québec et à travers le monde. Le Partenaire. $2012 ; 21(1): 4$
30.Larose-Hébert K. Quand les mots se heurtent aux maux : Portait d'un discours morcelé. Étude de l'expérience subjective des personnes utilisatrices des services de santé mentale au Québec [Thèse]. Ottawa (ON) : Université d'Ottawa ; 2016.

31.Parron A. Les enjeux de l'autonomisation de jeunes adultes confrontés à des troubles psychiques. Alter - European Journal of Disability Research. $2014 ; 8: 5$.

32.Gaudet S. L'émergence de l'âge adulte, une nouvelle étape du parcours de vie. Implications pour le développement de politiques. Document de discussion. [En ligne]. Ottawa: Gouvernement du Canada : Projet de recherche sur les politiques ; 2007 [consulté le 19 septembre 2018]. Disponible: http://horizons.gc.ca/sites/default/files/Publication-altformat/dp_youth_gaudet_200712_f.pdf.

33.Gagné J, Poirier M. Formes de l'appauvrissement et insertion sociale des jeunes adultes psychiatrisés. La réinsertion sociale. $1988 ; 13(1)$ : 132-43.

34.Fleury MJ, Grenier G. Parcours en milieu ordinaire de jeunes avec un handicap mental ou psychique. Santé Publique. 2013; 25 (4) : 460.

35.Turcotte ME, Bellot C. Vers une meilleure compréhension de la contribution des services sociaux à l'insertion sociale de jeunes adultes en difficulté. Société et jeunesse en difficulté. $2009 ; 8$.

36.Beau R. Les jeunes adultes hospitalisés en psychiatrie, un problème à repenser. Santé mentale au Québec. 1982 ; 7(1): 42-9.

37.Gagné J, Poirier M. Formes de l'appauvrissement et insertion sociale des jeunes adultes psychiatrisés. La réinsertion sociale. 1988 ; 13(1) : 132-43.

38. Leclerc C. Adaptation à la communauté de jeunes adultes ayant vécu une ou plusieurs hospitalisations en psychiatrie. Santé mentale au Québec. 1996 ; 21(2) : 53-72.

39.Ouellet-Plamondon C, Dubreucq S, Bérard M, Daneault JG, L'Heureux S, Olivier D et coll. L'intervention précoce pour la psychose... au Québec et à travers le monde. Le Partenaire. 2012 ; 21(1) : 5.

40.Ministère de la Santé et des soins de longue durée (MSSLD). Esprit ouvert, esprit sain : Stratégie ontarienne globale de santé mentale et de lutte contre la dépendance. [En ligne]. Toronto : Ministère de la Santé et des soins de longue durée; 2011 [consulté le 19 septembre 2018]. Disponible : http:// www.health.gov.on.ca/fr/common/ministry/publications/ reports/mental_health2011/mentalhealth_rep2011.pdf. 
40.Kirby M, Goldbloom D, Bradley L. Changer des orientations changer des vies : Stratégie en matière de santé mentale pour le Canada. [En ligne]. Ottawa : Commission de santé mentale du Canada ; 2012 [consulté le 19 septembre 2018]. Disponible: https://www.mentalhealthcommission.ca/sites/ default/files/MHStrategy_Strategy_FRE_1.pdf.

41. Ministère de la Santé et des services sociaux (MSSS). Plan d'action en santé mentale 2005-2010 : La force des liens. [En ligne]. Québec : Ministère de la Santé et des services sociaux; 2005 [consulté le 19 septembre 2018]. Disponible: http://publications.msss.gouv.qc.ca/msss/ fichiers/2005/05-914-01.pdf.

42. Ministère de la Santé et des services sociaux (MSSS). Plan d'action en santé mentale 2015-2020 : Faire ensemble et autrement. [En ligne]. Québec : Ministère de la Santé et des services sociaux ; 2015 [consulté le 19 septembre 2018]. Disponible: http://publications.msss.gouv.qc.ca/msss/ fichiers/2015/15-914-04W.pdf.

43.Boivert A. Résidence Paul-Pau, passage en douceur vers un projet de vie adulte. Le Partenaire. 2012 ; 21(1) : 9-12.

44.Cappelli M, Davidson S, Racek J, Leon S, Vloet M, Tataryn $\mathrm{K}$ et coll. Transitioning Youth into Adult Mental Health and Addiction Services: An Outcomes Evaluation of the Youth Transition Project. The Journal of Behavioral Health Services \& Research. 2016 ; 43(4) : 597-610.

45.Singh SP, Tuomainen $\mathrm{H}$. Transition From Child to Adult Mental Health Services: Needs, Barriers, Experiences and New Models of Care. World Psychiatry. 2015 ; 14(3) : 358-61.

46.Davis M. Addressing the Needs of Youth in Transition to Adulthood. Administrative and Policy in Mental Health. 2003 ; 30(6) : 495-509.

47.Davis M. Addressing the Needs of Youth in Transition to Adulthood. Administrative and Policy in Mental Health. 2003; 30(6) : 495-509.

48.Cappelli M, Davidson S, Racek J, Leon S, Vloet M, Tataryn $\mathrm{K}$ et coll. Transitioning Youth into Adult Mental Health and Addiction Services: An Outcomes Evaluation of the Youth Transition Project. The Journal of Behavioral Health Services \& Research. 2016 ; 43(4) : 597-610.

49.Singh SP, Tuomainen $\mathrm{H}$. Transition From Child to Adult Mental Health Services: Needs, Barriers, Experiences and New Models of Care. World Psychiatry. 2015 ; 14(3) : 358-61.

50.Davis M. Addressing the Needs of Youth in Transition to Adulthood. Administrative and Policy in Mental Health. 2003 ; 30(6) : 495-509.
51.Davis M. Addressing the Needs of Youth in Transition to Adulthood. Administrative and Policy in Mental Health. 2003; 30(6) : 495-509.

52.Gaudet S. L'émergence de l'âge adulte, une nouvelle étape du parcours de vie. Implications pour le développement de politiques. Document de discussion. [En ligne]. Ottawa: Gouvernement du Canada : Projet de recherche sur les politiques ; 2007 [consulté le 19 septembre 2018]. Disponible: http://horizons.gc.ca/sites/default/files/Publication-altformat/dp_youth_gaudet_200712_f.pdf.

53.Wilson CJ, Rickwood DJ, Bushnell JA, Caputi, P, Thomas SJ. The Effects of Need for Autonomy and Preference for Seeking Help from Informal Sources on Emerging Adults' Intentions to Access Mental Health Services for Common Mental Disorders and Suicidal Thoughts. Advances in Mental Health. 2011; 10: 29-38.

54.Gulliver A, Griffiths KM, Christensen H. Perceived barriers and facilitators to mental health help-seeking in young people: A systematic review. BMC Psychiatry. $2010 ; 10$.

55.Larose-Hébert K. Quand les mots se heurtent aux maux : Portait d'un discours morcelé. Étude de l'expérience subjective des personnes utilisatrices des services de santé mentale au Québec [Thèse]. Ottawa (ON) : Université d'Ottawa ; 2016.

Pour contacter les auteurs:

Alexis H Truong Ph.D.

Professeur adjoint

Université d'Ottawa

Département de criminologie

120 Université

Ottawa, Ontario K1N 6N5

Canada

couriel : ah.truong@uottawa.ca

Geneviève Nault

Coordonnatrice de stage et de la formation pratique

Université d'Ottawa

Département de criminologie

Katharine Larose-Hébert

Professeur adjoint

Université Laval

École de service social et de criminologie 